\title{
Some Extensions on Numbers
}

\section{Balasubramani Prema Rangasamy}

Former Student of Ramanujan Institute for Advanced Study in Mathematics, University of Madras, Chennai, India

Email: balguve@gmail.com

How to cite this paper: Rangasamy, B.P. (2019) Some Extensions on Numbers. $A d$ vances in Pure Mathematics, 9, 944-958.

https://doi.org/10.4236/apm.2019.911047

Received: October 23, 2019

Accepted: November 26, 2019

Published: November 29, 2019

Copyright $\odot 2019$ by author(s) and Scientific Research Publishing Inc. This work is licensed under the Creative Commons Attribution International License (CC BY 4.0).

http://creativecommons.org/licenses/by/4.0/

\begin{abstract}
My previous work dealt finding numbers which relatively prime to factorial value of certain number, high exponents and also find the way for finding mod values on certain number's exponents. Firstly, I retreat my previous works about Euler's phi function and some works on Fermat's little theorem. Next, I construct exponent parallelogram to find coherence numbers of Euler's phi functioned numbers and apply to Fermat's little theorem. Then, I test the primality of prime numbers on Pascal's triangle and explore new ways to construct Pascal's triangle. Finally, I find the factorial value for certain number by using exponent triangle.
\end{abstract}

\section{Keywords}

Factorial, Fermat's Little Theorem, Fermat's Last Theorem, Euler's Totient Function, Totient Function of $n$th Factorial, Totient Function of $n$th Exponent, Division on Exponents, Prime Bases on Fermat's Last Theorem, Exponent Parallelogram, Addition Triangle, Difference Triangle, Multiplication Triangle, Division Triangle, Exponent Triangle

\section{Introduction}

We know Fermat's little theorem and Euler's $\varphi$ (phi) function. Such are well defined operations on number theory and algebra. Euler's $\varphi$ (phi) function is considered as general proof of Fermat's little theorem. We seek other ways to find mod values on Fermat's little theorem, and generalize $\varphi$ (phi) function for a certain integer's exponentiation and factorial value. We construct the exponent parallelogram to find the coherence values of Euler's $\varphi$ (phi) function. We find higher valued exponents on Fermat's little theorem according to this. We also specify Fermat's last theorem by using prime numbers. Also we know binomial coefficients are constructing Pascal's triangle, in which we see 
the divisibility of prime numbers (primality test) in prime number exponentiation on Pascal's triangle. In addition, we construct Pascal's triangle and seek other ways except for binomial coefficients, i.e. and construct Pascal's triangle by arithmetic operations triangle. Finally instead of binomial coefficients in Pascal' triangle, we use exponents value of certain integer to construct Pascal's triangle, and then use " $n$ "th expansion to find factorial of such certain number.

First Blaise Pascal (1623-1662) introduced Pascal's triangle, after that, Isaac Newton (1643-1727) used the facts of Pascal's triangle he developed binomial expansion. He and his followers used binomial theorem for Probability and Statistical problems. Factorial were used to count permutations at as early as the $12^{\text {th }}$ century, by Indian scholars. In 1677, Fabian Stedman described factorial as applied to change ringing, a musical art involving the ringing of many tuned bells. In his words "Now the nature of these methods is such that the change of one number comprehends (includes) changes on lesser numbers". In that mean period, James Stirling (1692-1770) first introduced one approximation for finding $n$th factorial of a certain number. Then Adrien-Marrie Legendre used Leonhard Euler's (1707-1783) second integral formula and notated a symbol for it and then named it as Gamma function. It was a good approximation finding factorial of Real numbers. Jacques Philippe Marie Binet (1786-1856), modified James Stirling's approximation. Finally, the notation $n$ ! was introduced by the French mathematician Christian Kramp in 1808. Pierre de Fermat (1601-1665) stated Little theorem, for any two relatively prime numbers, in which exponent should be prime number; after that Leonhard Euler (1707-1783) found Totient function and then generalized Fermat's little theorem for any two relatively prime numbers.

From this book "Prime numbers a computational Perspective" [1], we know prime numbers and primality test. From this paper "Fermat's little theorem" [2], we know various types of explanations about Fermat's little theorem.

Prepositions 2 to 6 are worked by me. They are noted as PRB which means Prema. R. Balasubramani [3]. They are published in Fermat's theorem one extension: Mathematical Sciences International Journal ISSN 2278-8697 VOLUME 8 ISSUE 1 (JUNE 2019), P. 6-10.

In this paper,

1) I retreat my previous work Fermat's theorem one extension. Here I extend my works to finding the coherence numbers (constructing exponent parallelogram) for Euler's phi function and then generalize it for Fermat's little theorem.

2) I test the primality of prime numbers on Pascal's triangle.

3) I specify Fermat's last theorem by prime numbers.

4) I generate Pascal's triangle by arithmetic operations.

5) I find factorial value for certain number by using exponent triangle. 


\section{Discussion and Results}

Hint 1: Define $G_{p}=\{r \in N \mid 0<r<p n$ and $\operatorname{gcd}(n, r)=1\}$. Then $\left|G_{p}\right|=p \Phi(n)$.

Hint 2: Define $G_{p}=\{r \in N \mid(p-1) n<r<p n$ and $\operatorname{gcd}(n, r)=1\}$. Then $|G|=(p-1) \Phi(n)$.

\subsection{Let's Now Examine $\varphi(\mathrm{pn})$ When $p$ Is a Factor of $n$}

Lemma 1: Let $p$ be a prime and $p$ divides $n$, then $\Phi(p n)=p \Phi(n)$.

Proof: Notice that all the numbers that are relatively prime to $p n$ are also relatively prime to $n$. since $\operatorname{gcd}(p n, n)=n$ and $p$ divides $n$ the following result follows: $\operatorname{gcd}(n, r)=1$ if and only if $\operatorname{gcd}(p n, r)=1$ for any natural number $r$.

There are $p$ intervals, each with $\Phi(n)$ numbers relatively prime to $p n$, hence by the hint 1: the set $G_{p}=\{r \in N \mid 0<r<p n$ and $\operatorname{gcd}(n, r)=1\}$ has $\left|G_{p}\right|=p \Phi(n)$ elements.

For our example we choose 20 , so let's consider $2 \times 20$; $\Phi(2 \times 20)=2 \Phi(20)=2 \times 8=16$. Putting together the two sets mentioned in our previous examples we have $\{1,3,7,9,11,13,17,19,21,23,27,29,31,33,37,39\}$, exactly all 16 numbers are relatively prime to 40 .

\subsection{Let's Now Examine $\varphi(\mathrm{pn})$ When $\boldsymbol{p}$ Is Not a Factor of $\boldsymbol{n}$}

Lemma 2: Let $p$ be a prime and $p$ does not divide $n$, then $\Phi(p n)=(p-1) \Phi(n)$.

Proof: We know that $p \Phi(n)$ is the number of numbers relatively prime to $n$ and less than $p n$. Notice that all the multiples of $p$ whose factors are relatively prime to $n$ are counted, since $\operatorname{gcd}(p, n)=1$. Notice the conditions imply $\operatorname{gcd}(p n, r)=1$ iff $\operatorname{gcd}(n, r)=1$ and $\operatorname{gcd}(p, r)=1$.

Suppose the list of multiples is $\left\{r_{1} p, r_{2} p, r_{3} p, \cdots, r_{\Phi(n)} p\right\}$, where all the $r^{2}$ 's are relatively prime to $n$. the set has $\Phi(n)$ numbers relatively prime to $n$ and 0 relatively prime to $p$, because they are all multiples of $p$. we subtract this many from our original count and we have $\Phi(p n)=p \Phi(n)-\Phi(n)=(p-1) \Phi(n)$. $\square$

For our examples we choose 20 , so let's consider $3 \times 20$; $\Phi(3 \times 20)=(3-1) \Phi(20)=2 \times 8=16$. Putting together the two sets mentioned in our previous examples we have $\{1,7,11,13,17,19,23,29,31,37,41,43,47$, $49,53,59\}$ exactly all 16 numbers relatively prime to 60 .

Preposition 1: Let $n$ be a positive integer. Then $\varphi(n !)=n \varphi((n-1) !)$ when $n$ is composite number and $\varphi(n !)=(n-1) \varphi((n-1) !) \quad$ when $n$ is prime number.

Proof: Let $\mathrm{n}$ be a positive integer.

When $n$ be a composite number and $n$ divides $(n-1) !$, then $\varphi(n !)=\varphi[n(n-1) !]=n \varphi[(n-1) !]$.

Notice that all the numbers that are relatively prime to $n(n-1) !$ are also relatively prime to $(n-1)$ !. Since $\operatorname{gcd}[n(n-1) !,(n-1) !]=(n-1)$ ! And $n$ divides 
$(n-1)$ ! The following result follows: $\operatorname{gcd}[(n-1) !, r]=1$ if and only if $\operatorname{gcd}[n(n-1) !, r]=1$ for any natural number $r$.

There are $n$ intervals, each with $\varphi((n-1) !)$ numbers relatively prime to $n(n-1)$ ! , hence by the hint 1 : the set

$G_{n}=\{r \in N \mid 0<r<n(n-1) !$ and $\operatorname{gcd}((n-1) !, r)=1\} \quad$ has $\quad\left|G_{n}\right|=n \varphi((n-1) !)$ elements.

When $n$ be a prime and $\mathrm{n}$ does not divide $(n-1)$ !, then $\varphi(n !)=(n-1) \varphi[(n-1) !]$.

We know that $n \varphi((n-1) !)$ is the number of numbers relatively prime to $(n-1)$ ! and less than $n(n-1)$ !. Notice that all the multiples of $n$ whose factors are relatively prime to $(n-1) !$ are counted, since $\operatorname{gcd}[n,(n-1) !]=1$. Notice the conditions imply $\operatorname{gcd}[n(n-1) !, r]=1$ if and only if $\operatorname{gcd}[(n-1) !, r]=1$ and $\operatorname{gcd}(n, r)=1$.

Suppose the list of multiples is $\left\{r_{1} n, r_{2} n, r_{3} n, \cdots, r_{\varphi((n-1) !)} n\right\}$, where all the $r^{2}$ s are relatively prime to $(n-1)$ !. The set has $\varphi((n-1) !)$ numbers relatively prime to $(n-1)$ ! and 0 relatively prime to $n$, because they are all multiples of $n$. by this way we get $\varphi(n !)=\varphi[n(n-1) !]=\varphi(n) \varphi[(n-1) !]=(n-1) \varphi[(n-1) !]$.

Preposition 2 (PRB): Let $n$ be a positive integer. Then $\varphi(n !)=\prod n_{i}(n-1)_{i} \varphi(2)$ where $n_{i}$ 's are composite numbers and $(n-1)_{i}$ 's are prime numbers not exceeding $n$.

\section{Proof:}

Using preposition 1 , we obtained $\varphi(n !)=n \varphi((n-1) !)$ when $n$ is composite number and

$\varphi(n !)=(n-1) \varphi[(n-1) !]$ when $n$ is prime number. Since all even numbers are composites except 2 because 2 is prime. So we cannot find an even composite number less than four. And two is the only prime number less than three. Also 1 is the only number relatively prime to two and below it. So we obtained from these two equations we get

$$
\varphi(n !)=\prod n_{i}(n-1)_{i} \varphi(2)
$$

Example 1: Find the value of $\varphi(8 !)$

\section{Solution:}

Let $\varphi(8 !)$ then we can write $\varphi(8 !)=\varphi(8 \times 7 !)$. So

$$
\varphi(8 \times 7 !)=8 \varphi(7 \times 6 !)=8 \varphi(7) \varphi(6 !)=8 \times 6 \times \varphi(6 !)=8 \times 6 \times 192=9216 .
$$

Example 2: Find the value of $\varphi(13$ !)

\section{Solution:}

Let $\varphi(13 !)$ then $\varphi(13 !)=\varphi(13 \times 12 !)$. So

$$
\begin{aligned}
\varphi(13 \times 12 !) & =12 \varphi(12 !) \\
& =12 \times 12 \times 10 \times 10 \times 9 \times 8 \times 6 \times 6 \times 4 \times 4 \times 2 \times \varphi(2) \\
& =1194393600
\end{aligned}
$$


Preposition 3 (PRB): Let $n$ be a positive integer and " $a$ " be an exponent to $n$. Then $\varphi\left(n^{a}\right)=n^{a-1} \varphi(n)$.

Proof: The positive integers less than $n^{a}$ that are not relatively prime to $n$ are those integers not exceeding $n^{a}$ that are divisible by $n$. There are exactly $n^{a-1}$ such integers, so there are $n^{a-1} \varphi(n)$ integers less than $n^{a}$ that are relatively prime to $n^{a}$.

Hence,

$$
\varphi\left(n^{a}\right)=n^{a-1} \varphi(n)
$$

Example 4: Find the value of $\varphi\left(10^{4}\right)$.

\section{Solution:}

Let $\varphi\left(10^{4}\right)$ then $\varphi\left(10^{4}\right)=\varphi\left(10^{3} \times 10\right)=10^{3} \varphi(10)=1000 \times 4=4000$.

Since 10 is a composite, $10^{4}=10,000$ so $\varphi(10,000)=4000$.

Example 5: Find the value of $\varphi\left(331^{5}\right)$.

\section{Solution:}

Let $\varphi\left(331^{5}\right)$ then

$$
\begin{aligned}
\varphi\left(331^{5}\right) & =\varphi\left(331^{4} \times 331\right)=331^{4} \varphi(331) \\
& =12003612721 \times 330=3961192197930
\end{aligned}
$$

Since 331 is a prime, $331^{5}=3,973,195,810,651$ so $\varphi(3,973,195,810,651)=$ $3,961,192,197,930$.

\subsection{Exponent Division on Fermat's Little Theorem}

Preposition 4 (PRB): If $p$ is prime and " $a$ " is a positive integer with $p$ does not divides " $a$ ", $\operatorname{gcd}(a, p)=1$ and $n$ be an exponent to " $a$ " then $a^{n} \equiv r^{s+t}(\bmod p) . r$ is a congruent of " $a$ " for $\bmod p$, where " $s$ " is a quotient and " $t$ " is a residue when " $n$ " divided by $\mathrm{p}$ and $n \in \boldsymbol{N}$ is any exponent.

Proof: Let $p$ be a prime, and $a$ is a positive integer with $p$ does not divides $a$, $\operatorname{gcd}(a, p)=1$ and $n$ be an exponent to $a$ then

$a^{n} \equiv(q p+r)^{n} \equiv r^{n}(\bmod p) ; q \in Z$

$r^{n} \bmod p=r^{s_{1} p+t_{1}} \equiv r^{s_{1}+t_{1}}(\bmod p)$ if $s_{1}+t_{1} \geq p$ then

$r^{s_{1}+t_{1}} \equiv r^{s_{2} p+t_{2}} \equiv r^{s_{2}+t_{2}}(\bmod p)$ if $s_{2}+t_{2} \geq p$ then

Do this again and again until we get $s_{k}+t_{k}<p, k \in N$

$$
r^{s_{k-1}+t_{k-1}} \equiv r^{s_{k} p+t_{k}} \equiv r^{s_{k}+t_{k}}(\bmod p) \text { if } s_{k}+t_{k}<p .
$$

Hence we get,

$$
a^{n} \equiv r^{s+t}(\bmod p)
$$

\subsection{Proving Fermat's Little Theorem, Using Preposition 4}

If $p$ is prime and $a$ is a positive integer with $p$ does not divides " $a$ " and $\operatorname{gcd}(a, p)=1$ then $a^{p-1} \equiv 1(\bmod p)$.

$\Rightarrow a^{p-1} \equiv a^{(1+0)-1} \equiv a^{1-1} \equiv a^{0} \equiv 1(\bmod p)$.

Example 6: Find the value of $3^{1900} \bmod 13$. 
Solution: We can write

$1900=146.13+2$

$\equiv 146+2=148$ here $148 \geq 13$ so,

$148=11.13+5$

$\equiv 16$ here $16 \geq 13$ so,

$19=1.13+3$

$\equiv 4$ here $4<13$ so,

Apply this algorithm, then we get

$$
3^{1900} \equiv 3^{4}=81 \equiv 3 \bmod 13 .
$$

Preposition 5 (PRB): If $m$ is a positive integer and $a$ is an integer with $(a, m)$ $=1$,

Then $a^{[\varphi(m)+w] !} \equiv 1(\bmod m)$.

where

$$
w \in W
$$

\section{Proof:}

Let $a^{[\varphi(m)+w] !}=a^{k}$. So we can write $k=n \varphi(m)+k^{\prime}$ for some integer $m$. now we can write $a^{k}=a^{n \varphi(m)}$. Here $k^{\prime}=0$, since $k$ value has $\varphi(m)$ as a one factor and $n$ is a positive integer.

It gives

$$
\left(a^{\varphi(m)}\right)^{n} \equiv 1^{n} \equiv 1(\bmod m)
$$

Example 8: Find the value of $5^{[\varphi(9)+5] !} \bmod 9$

\section{Solution:}

$$
5^{[\varphi(9)+5] !}=5^{[6+5] !}=5^{11 !} \equiv\left[5^{6}\right]^{6652800} \equiv[1]^{6652800} \equiv 1 \bmod 9 .
$$

Preposition 6 (PRB): If $m$ is a positive integer and $a$ is an integer with $(a, m)$ $=1$,

Then $a^{\varphi(m)^{n}} \equiv 1(\bmod m)$.

where

$$
n \in N
$$

Proof: Let $[\varphi(m)]^{n}=k$ then $a^{\varphi(m)^{n}}=a^{k} \bmod m$. So we can write $k=h \varphi(m)+k^{\prime}$ for some integer $m$. Now $a^{k}=a^{h \varphi(m)}$. Here $k^{\prime}=0$. Since $k$ has $\varphi(m)$ as a one factor and $h$ is a positive integer. It gives $\left(a^{\varphi(m)}\right)^{h} \equiv 1^{h} \equiv 1(\bmod m)$.

\subsection{Exponent Parallelogram}

Definition 1: Let $m \in Z-\{0\}$ and $n \in Z$ be the exponent to $m$ then do $1^{\text {st }}$ operation is subtracting each element with its successive element of $1^{\text {st }}$ line elements. Result will be $(m-1) m^{n}$, we shall name $(m-1)$ as “ $a$ ". $2^{\text {nd }}$ operation is subtracting each element with its successive element of $1^{\text {st }}$ operation, result will be $(m-1)^{2} m^{n}$ then we shall name $(m-1)^{2}$ as " $a^{2 \text { ". }} 3^{\text {rd }}$ operation is subtracting 
each element with its successive element of $2^{\text {nd }}$ operation, result will be

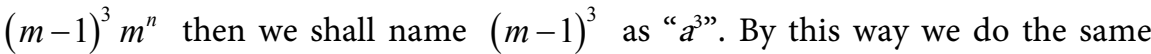
up to $n$th operation. These $1^{\text {st }}$ line to $n$th operation diagonal elements coefficients construct exponent parallelogram.

Now we construct exponent parallelogram:

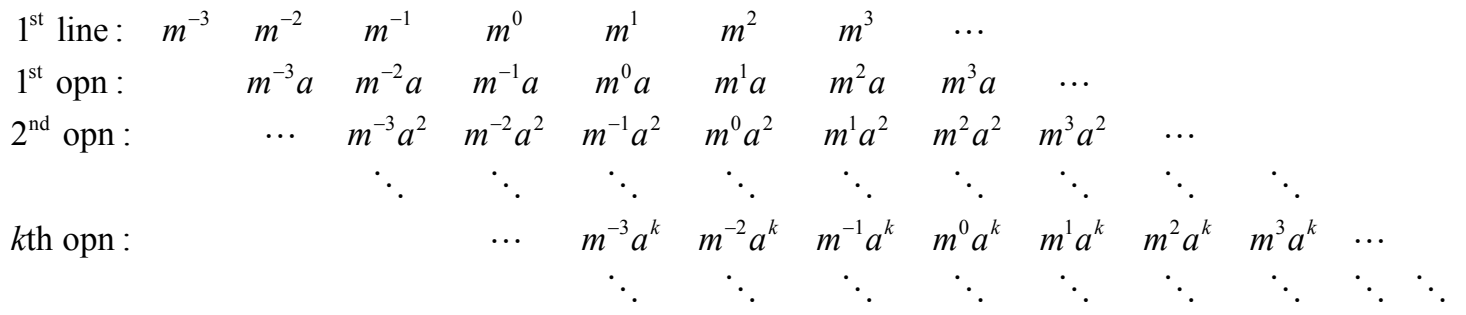

Note: $m^{n} a^{k}$ should be placed between $m^{n} a^{k-1}$ and $m^{n+1} a^{k-1}$ in $k$ th operation. Because $m^{n} a^{k}=m^{n+1} a^{k-1}-m^{n} a^{k-1}$.

Let we construct exponent plane for 5: for $m, k \in I$ and $n \in Z$

$$
\begin{aligned}
& \begin{array}{lllllllll}
\cdots & 5^{-3} & 5^{-2} & 5^{-1} & 5^{0} & 5^{1} & 5^{2} & 5^{3} & \cdots
\end{array} \\
& \begin{array}{lllllllll}
\cdots & 5^{-3} 4 & 5^{-2} 4 & 5^{-1} 4 & 5^{0} 4 & 5^{1} 4 & 5^{2} 4 & 5^{3} 4 & \cdots
\end{array} \\
& \begin{array}{lllllllll} 
& 5^{-3} 4^{2} & 5^{-2} 4^{2} & 5^{-1} 4^{2} & 5^{0} 4^{2} & 5^{1} 4^{2} & 5^{2} 4^{2} & 5^{3} 4^{2} & \ldots
\end{array}
\end{aligned}
$$

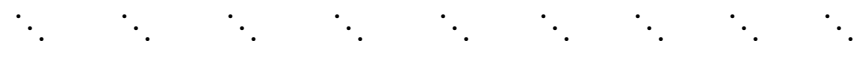

$$
\begin{aligned}
& \begin{array}{lllllllll}
\ldots & 5^{-3} 4^{k} & 5^{-2} 4^{k} & 5^{-1} 4^{k} & 5^{0} 4^{k} & 5^{1} 4^{k} & 5^{2} 4^{k} & 5^{3} 4^{k} & \ldots
\end{array}
\end{aligned}
$$

Now we get,

$$
\begin{array}{cccccccccccc}
\cdots & \frac{1}{125} & \frac{1}{25} & \frac{1}{5} & 1 & 5 & 25 & 125 & \cdots & & & \\
\cdots & \frac{4}{125} & \frac{4}{25} & \frac{4}{5} & 4 & 20 & 100 & 500 & \cdots & & & \\
& \cdots & \frac{16}{125} & \frac{16}{25} & \frac{16}{5} & 16 & 80 & 400 & 2000 & \cdots & & \\
& & \ddots & \ddots & \ddots & \ddots & \ddots & \ddots & \ddots & \ddots & \ddots & \\
& & & \cdots & 5^{-3} 4^{k} & 5^{-2} 4^{k} & 5^{-1} 4^{k} & 5^{0} 4^{k} & 5^{1} 4^{k} & 5^{2} 4^{k} & 5^{3} 4^{k} & \cdots
\end{array}
$$

By the above results we define,

1) If " $E$ " is a $1^{\text {st }}$ line prime exponent and " $a$ " is an integer with $(a, E)=1$, then $a^{E} \equiv a(\bmod E)$.

2) If " $E$ " is a prime exponent and " $a$ " is an integer with $(a, E)=1$, then $a^{E[\varphi(E)] k} \equiv 1(\bmod E)$, where " $k$ " is any positive integer of $1^{\text {st }}$ operation to $k$-th operation coherence numbers of $\varphi(E)$.

\section{Examples:}

1) Let 7 is a first line prime exponents i.e. $(1,7,49,343, \ldots)$ and $4 \in I$ with $(4,7)=1$, then $4^{(1,7,49,343, \cdots)} \equiv 4(\bmod 7)$.

2) Let 7 is a first line prime exponents, $(6,42,294,2058, \ldots)$ are $1^{\text {st }}$ operation to $k$ th operation and $4 \in I$ with $(4,7)=1$, then $4^{(6,42,294,2058, \cdots)} \equiv 1(\bmod 7)$. Where $6,42,294,2058, \cdots$ are coherence numbers of $\varphi(7)$. 
3) Let 5 is a first line prime exponents, $(4,5 \times 4,25 \times 4,125 \times 4, \cdots, 16,5 \times$ $16,25 \times 16, \cdots, 64,5 \times 64,25 \times 64, \cdots)$ are $1^{\text {st }}$ operation to $k$ th operation and $4 \in I \quad$ with $(4,5)=1$, then $4^{5[\varphi(5)] k} \equiv 1, \quad 4^{25[\varphi(5)] k} \equiv 1, \quad 4^{125[\varphi(5)] k} \equiv 1, \cdots$, $4^{5^{n}[\varphi(5)] k} \equiv 1(\bmod 5)$. Where $(4,5 \times 4,25 \times 4,125 \times 4, \cdots, 16,5 \times 16,25 \times 16$, $\cdots, 64,5 \times 64,25 \times 64, \cdots)$ are coherence numbers of $\varphi(5)$ and $k, n \in I$.

\subsection{Prime Bases on Fermat's Last Theorem}

Let we see following summations.

Let $p_{i}$ are prime numbers then

$2+3+5+7+11+13=41$;

\section{For squared primes:}

$$
\begin{aligned}
& 4+9=13=(3.6055 \cdots)^{2}=(3+0.6055 \cdots)^{2} ; \\
& 4+9+25=38=(6.1644 \cdots)^{2}=(5+1.1644 \cdots)^{2} ; \\
& 4+9+25+49=87=(9.3273 \cdots)^{2}=(7+2.3273 \cdots)^{2} ; \\
& 4+9+25+49+121=208=(14.4222 \cdots)^{2}=(11+3.4222 \cdots)^{2} ;
\end{aligned}
$$

\section{For cubed primes:}

$$
\begin{aligned}
& 8+27=35=(3.2710 \cdots)^{3}=(3+0.2710 \cdots)^{3} ; \\
& 8+27+125=160=(5.4288 \cdots)^{3}=(5+0.4288 \cdots)^{3} ; \\
& 8+27+125+343=503=(7.9528 \cdots)^{3}=(7+0.9528 \cdots)^{3} ; \\
& 8+27+125+343+1331=1724=(12.2405 \cdots)^{3}=(11+1.2405 \cdots)^{3} ;
\end{aligned}
$$

For fourth exponent primes:

$$
\begin{aligned}
& 16+81=97=(3.1382 \cdots)^{4}=(3+0.1382 \cdots)^{4} \\
& 16+81+625=722=(5.1836 \cdots)^{4}=(5+0.1836 \cdots)^{4} \\
& 16+81+625+2401=3123=(7.4755 \cdots)^{4}=(7+0.4755 \cdots)^{4} ; \\
& 16+81+625+2401+14641=17764=(11.5447 \cdots)^{4}=(11+0.5447 \cdots)^{4}
\end{aligned}
$$

By this way we concluded,

$$
2^{n}+3^{n}+5^{n}+7^{n}+11^{n}+13^{n}+\cdots+p_{i}^{n}=\left(p_{i}+B \cdot b_{1} b_{2} b_{3} b_{4} \cdots\right)^{n} \text {. }
$$

where $B . b_{1} b_{2} b_{3} b_{4} \cdots \in R$.

From the above recursion, we formulate the result then we get,

$$
\sum_{n=2}^{k} p_{i}^{n}=\left(p_{i}+B \cdot b_{1} b_{2} b_{3} b_{4} \cdots\right)^{n} \text {. }
$$

where

$$
B . b_{1} b_{2} b_{3} b_{4} \cdots \in R
$$

Theorem 1: Let $p_{i}$ are prime numbers then $\sum_{n=2}^{k} p_{i}^{n} \neq q^{n}$. Where $q$ is any prime.

Proof:

Let $\sum_{n=2}^{k} p_{i}^{n}=P$ then

Case 1: If $P$ is prime, result is obvious.

Case 2: If $P$ is composite, we can write $P=s t+k$. if $k \neq 0$ then result is obvious.

Case 3: If $P$ is composite and $k=0$, then we can write $P=s t$. If $s, t$ are 
$1^{\text {st }}$ line:

$1^{\text {st }}$ operation:

$2^{\text {nd }}$ operation:

$3^{\text {rd }}$ operation:

$4^{\text {th }}$ operation:

$5^{\text {th }}$ operation: distinct primes then result is obvious. But if $s=t$ we get $\sum_{n=2}^{k} p_{i}^{n}=q^{n}$. This result contradict with (1). So $\sum_{n=2}^{k} p_{i}^{n} \neq q^{n}$. Where $q$ is any prime.

\subsection{Primality of Pascal's Triangle}

Definition 2: For all $n \in N$ and for all reals $a, b \in R$ we have the formula $(a+b)^{n}=\sum_{k=0}^{n}\left(\begin{array}{l}n \\ k\end{array}\right) a^{n-k} b^{k}$. For every $p=n$ we have $(a+b)^{p}=\sum_{k=0}^{p}\left(\begin{array}{l}p \\ k\end{array}\right) a^{p-k} b^{k}$ then $p$ divides $\sum_{k=1}^{p}\left(\begin{array}{l}p \\ k\end{array}\right)$ and every $\left(\begin{array}{l}p \\ k\end{array}\right)$; where $k=1,2, \cdots, p-1$ is called Primality of binomial expansion.

\section{Prime number Pascal's triangle coefficients}

$\begin{array}{lllllllll}0 & 1 & & & & & & \\ 1 & 1 & 1 & & & & & & \\ 2 & 1 & 2 & 1 & & & & & \\ 3 & 1 & 3 & 3 & 1 & & & & \\ 4 & 1 & 4 & 6 & 4 & 1 & & & \\ 5 & 1 & 5 & 10 & 10 & 5 & 1 & & \\ 6 & 1 & 6 & 15 & 20 & 15 & 6 & 1 & \\ 7 & 1 & 7 & 21 & 35 & 35 & 21 & 7 & 1 \\ \ldots & & & & & & & & \\ 11 & 1 & 11 & 55 & 165 & 330 & 462 & 462 & 330 \ldots 1 \\ \ldots & & & & & & & \\ p & \left(\begin{array}{l}p \\ 0\end{array}\right)\left(\begin{array}{l}p \\ 1\end{array}\right)\left(\begin{array}{l}p \\ 2\end{array}\right) \ldots & \left(\begin{array}{l}p \\ k\end{array}\right) \text { where } 0 \leq k \leq p\end{array}$

\section{Examples:}

1) 7 divides $7+21+35+35+21+7$ i.e. $126 / 7=18$

2) 11 divides $2(11+55+165+330+462)$ i.e. $2046 / 11=186$.

\subsection{Constructing Pascal's Triangle by Arithmetic Triangles}

\section{Addition triangle}

Definition 3: Let $A, B, C, \cdots \in Z$ then do $1^{\text {st }}$ operation is adding each element with its successive element of $1^{\text {st }}$ line elements, $2^{\text {nd }}$ operation is adding each element with its successive element of $1^{\text {st }}$ operation, and 3 rd operation is adding each element with its successive element of 2 nd operation. By this way we do the same up to $n$th operation. These $1^{\text {st }}$ line to $n$th operation diagonal elements coefficients construct Pascal's triangle.

Now we construct addition triangle:

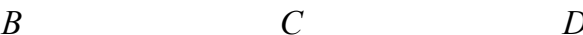

$$
\begin{aligned}
& B+A \\
& C+B \quad D+C \\
& E+D \\
& E+2 D+C \\
& F+2 E+D \\
& D+3 C+3 B+A \quad E+3 D+3 C+B \quad F+3 E+3 D+C \\
& E+4 D+6 C+4 B+A \quad F+4 E+6 D+4 C+B \\
& F+5 E+10 D+10 C+5 B+A
\end{aligned}
$$$$
D
$$$$
E
$$ 
From the above, using the colored diagonal we can construct a Pascal's triangle:

1

$1 \quad 1$

$\begin{array}{lll}1 & 2 & 1\end{array}$

$\begin{array}{llll}1 & 3 & 3 & 1\end{array}$

$\begin{array}{lllll}1 & 4 & 6 & 4 & 1\end{array}$

$\begin{array}{llllll}1 & 5 & 10 & 10 & 5 & 1\end{array}$

$\begin{array}{lllllll}1 & 6 & 15 & 20 & 15 & 6 & 1\end{array}$

$\left(\begin{array}{l}n \\ 0\end{array}\right) \quad\left(\begin{array}{l}n \\ 1\end{array}\right)\left(\begin{array}{l}n \\ 2\end{array}\right)\left(\begin{array}{l}n \\ 3\end{array}\right)-\cdots \quad-\left(\begin{array}{c}n \\ k-1\end{array}\right)\left(\begin{array}{l}n \\ k\end{array}\right)$.

\subsection{Backward Difference Triangle}

Definition 4: Let $A, B, C, \cdots \in Z$ then do $1^{\text {st }}$ operation is subtracting each element with its predecessor element of $1^{\text {st }}$ line elements, $2^{\text {nd }}$ operation is subtracting each element with its predecessor element of $1^{\text {st }}$ operation, and 3rd operation is subtracting each element with its predecessor element of 2 nd operation. By this way we do the same up to nth operation. These $1^{\text {st }}$ line to nth operation diagonal elements coefficients construct Pascal's triangle with negative coefficients.

Now we construct backward difference triangle:

$1^{\text {st }}$ line: $B$

C

D

E

F

$1^{\text {st }}$ operation:

$B-A$

$C-B$

$D-C$

$E-D$

$F-D$

$2^{\text {nd }}$ operation:

$C-2 B+$

$3^{\text {rd }}$ operation:

$4^{\text {th }}$ operation:

$5^{\text {th }}$ operation:

$$
\begin{gathered}
D-3 C+3 B-A \quad E-3 D+3 C-B \quad F-3 E+3 D-C \\
E-4 D+6 C-4 B+A \quad F-4 E+6 D-4 C+B \\
F-5 E+10 D-10 C+5 B-A
\end{gathered}
$$

From the above, using the colored diagonal we can construct a negative Pascal's triangle:

1

$-1 \quad 1$

$1 \quad-2 \quad 1$

$\begin{array}{llll}-1 & 3 & -3 & 1\end{array}$

$\begin{array}{lllll}1 & -4 & 6 & -4 & 1\end{array}$

$\begin{array}{llllll}-1 & 5 & -10 & 10 & -5 & 1\end{array}$

$\begin{array}{lllllll}1 & -6 & 15 & -20 & 15 & -6 & 1\end{array}$

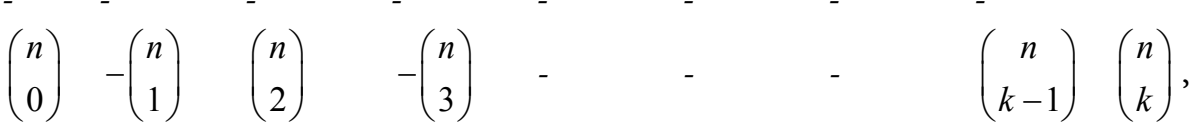

where $\left(\begin{array}{l}n \\ 0\end{array}\right)$ sign depends upon whether $n$ is odd or even. If $n$ is odd we get 
$-\left(\begin{array}{l}n \\ 0\end{array}\right)$, else we get $\left(\begin{array}{l}n \\ 0\end{array}\right)$.

\subsection{Forward Difference Triangle}

Definition 5: Let $A, B, C, \cdots \in Z$ then do $1^{\text {st }}$ operation is subtracting each element with its successive element of $1^{\text {st }}$ line elements, $2^{\text {nd }}$ operation is subtracting each element with its successive element of $1^{\text {st }}$ operation, and 3rd operation is subtracting each element with its successive element of 2nd operation. By this way we do the same up to nth operation. These $1^{\text {st }}$ line to nth operation diagonal elements coefficients construct Pascal's triangle with negative coefficients.

Now we construct forward difference triangle:

$A$

$B$

C

$D$

E

F

$$
\begin{array}{ccccc}
A-B & B-C & C-D & D-E & \\
A-2 B+C & B-2 C+D & C-2 D+E & D-2 E+F & \\
A-3 B+3 C-D & B-3 C+3 D-E & C-3 D+3 E-F & \\
A-4 B+6 C-4 D+E & B-4 C+6 D-4 E+F & \\
A-5 B+10 C-10 D+5 E-F &
\end{array}
$$

From the above, using the colored diagonal we can construct a negative Pascal's triangle:

1

$1-1$

$\begin{array}{lll}1 & -2 & 1\end{array}$

$\begin{array}{llll}1 & -3 & 3 & -1\end{array}$

$\begin{array}{lllll}1 & -4 & 6 & -4 & 1\end{array}$

$\begin{array}{llllll}1 & -5 & 10 & -10 & 5 & -1\end{array}$

$\begin{array}{lllllll}1 & -6 & 15 & -20 & 15 & -6 & 1\end{array}$

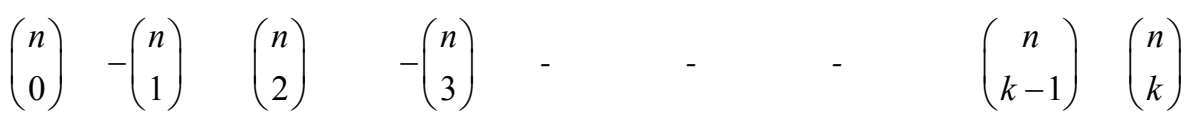
where $\left(\begin{array}{l}n \\ k\end{array}\right)$ sign depends upon whether $n$ is odd or even. If $n$ is odd we get $-\left(\begin{array}{l}n \\ k\end{array}\right)$, else we get $\left(\begin{array}{l}n \\ k\end{array}\right)$.

\subsection{Multiplication Triangle}

Definition 6: Let $A, B, C, \cdots \in Z$ then do $1^{\text {st }}$ operation is multiplying each element with its successive element of $1^{\text {st }}$ line elements, $2^{\text {nd }}$ operation is multiplying each element with its successive element of $1^{\text {st }}$ operation, and 3rd operation is multiplying each element with its successive element of 2nd operation. By this way we do the same up to nth operation. These $1^{\text {st }}$ line to nth operation diagonal elements degrees construct Pascal's triangle.

Now we construct multiplication triangle: 
A

$A B$

B

$A B^{2} C$
C

$B C$

$$
\begin{aligned}
& B C^{2} D \\
& A B^{3} C^{3} D \\
& A B^{4} C^{6} D^{4} E \\
& B C^{3} D^{3} E \\
& C D^{2} E
\end{aligned}
$$$$
B C \quad C D
$$

D

$D E$

E

F

EF $D E^{2} F$

$D E^{2} F$

From the above, using the colored diagonal exponents, we can construct a Pascal's triangle:

1

$1 \quad 1$

$\begin{array}{lll}1 & 2 & 1\end{array}$

$\begin{array}{lll}1 & 3 & 3\end{array}$

$\begin{array}{lll}1 & 4 & 6\end{array}$

$$
1
$$

4

$\left(\begin{array}{l}n \\ 0\end{array}\right) \quad\left(\begin{array}{l}n \\ 1\end{array}\right)$

$$
\left(\begin{array}{l}
n \\
2
\end{array}\right)
$$

$$
\left(\begin{array}{l}
n \\
3
\end{array}\right)
$$

5

15

\subsection{Forward Division Triangle}

Definition 7: Let $A, B, C, \cdots \in Z$ then do $1^{\text {st }}$ operation is dividing each element with its successive element of $1^{\text {st }}$ line elements, $2^{\text {nd }}$ operation is dividing each element with its successive element of $1^{\text {st }}$ operation, and 3rd operation is dividing each element with its successive element of 2nd operation. By this way we do the same up to nth operation. These $1^{\text {st }}$ line to nth operation diagonal elements degrees construct Pascal's triangle.

Now we construct forward division triangle:

$$
\begin{aligned}
& \begin{array}{lll}
A & B
\end{array} \\
& \frac{A}{B} \quad \frac{B}{C} \\
& \text { C } \\
& \frac{C}{D} \\
& \text { D } \\
& \frac{D}{E} \quad \frac{E}{F} \\
& \frac{A C}{B^{2}} \\
& \frac{B D}{C^{2}} \\
& \frac{A C^{3}}{B^{3} D} \\
& \frac{B D^{3}}{C^{3} E} \\
& \frac{C E}{D^{2}} \\
& \frac{D F}{E^{2}} \\
& \frac{A C^{6} E}{B^{4} D^{4}} \\
& \frac{C E^{3}}{D^{3} F} \\
& \frac{B D^{6} F}{C^{4} E^{4}} \\
& \frac{A C^{10} E^{5}}{B^{5} D^{10} F}
\end{aligned}
$$

From the above, using the colored diagonal exponents, we can construct a Pascal's triangle:

1

$1-1$

$1 \quad-2 \quad 1$

$\begin{array}{llll}1 & -3 & 3 & -1\end{array}$ 


$\begin{array}{llllllll}1 & -4 & 6 & -4 & 1 & & \\ 1 & -5 & 10 & -10 & 5 & -1 & \\ 1 & -6 & 15 & -20 & 15 & -6 & 1 & - \\ - & - & - & - & - & - & - & \left(\begin{array}{c}n \\ k-1\end{array}\right)\left(\begin{array}{l}n \\ k\end{array}\right),\end{array}$
where $\left(\begin{array}{l}n \\ k\end{array}\right)$ sign depends upon whether $n$ is odd or even. If $n$ is odd we get $-\left(\begin{array}{l}n \\ k\end{array}\right)$, else we get $\left(\begin{array}{l}n \\ k\end{array}\right)$.

Upon whether $n$ is odd or even. If $n$ is odd we get $\left(\begin{array}{l}n \\ k\end{array}\right)$, else we get $-\left(\begin{array}{l}n \\ k\end{array}\right)$.

\subsection{Backward Division Triangle}

Definition 8: Let $A, B, C, \cdots \in Z$ then do $1^{\text {st }}$ operation is dividing each element with its successive element of $1^{\text {st }}$ line elements, $2^{\text {nd }}$ operation is dividing each element with its successive element of $1^{\text {st }}$ operation, and 3rd operation is dividing each element with its successive element of 2 nd operation. By this way we do the same up to nth operation. These $1^{\text {st }}$ line to nth operation diagonal elements degrees construct Pascal's triangle.

Now we construct backward division triangle:

$$
\begin{aligned}
& \begin{array}{llll}
A & B & C
\end{array} \\
& \begin{array}{lllll}
\frac{B}{A} & \frac{C}{B} & \frac{D}{C} & \frac{E}{D} & \frac{F}{E}
\end{array} \\
& \frac{B^{2}}{A C} \quad \frac{C^{2}}{B D} \quad \frac{D^{2}}{C E} \quad \frac{E^{2}}{D F} \\
& \frac{B^{3} D}{A C^{3}} \quad \frac{C^{3} E}{B D^{3}} \quad \frac{D^{3} F}{C E^{3}} \\
& \frac{B^{4} D^{4}}{A C^{6} E} \quad \frac{C^{4} E^{4}}{B D^{6} F} \\
& \frac{B^{5} D^{10} F}{A C^{10} E^{5}}
\end{aligned}
$$

From the above, using the colored diagonal exponents, we can construct a Pascal's triangle:

$\begin{array}{lllllll}-1 & 6 & -15 & 20 & -15 & 6 & -1\end{array}$

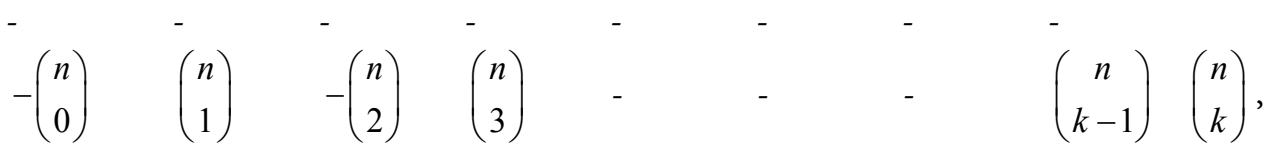




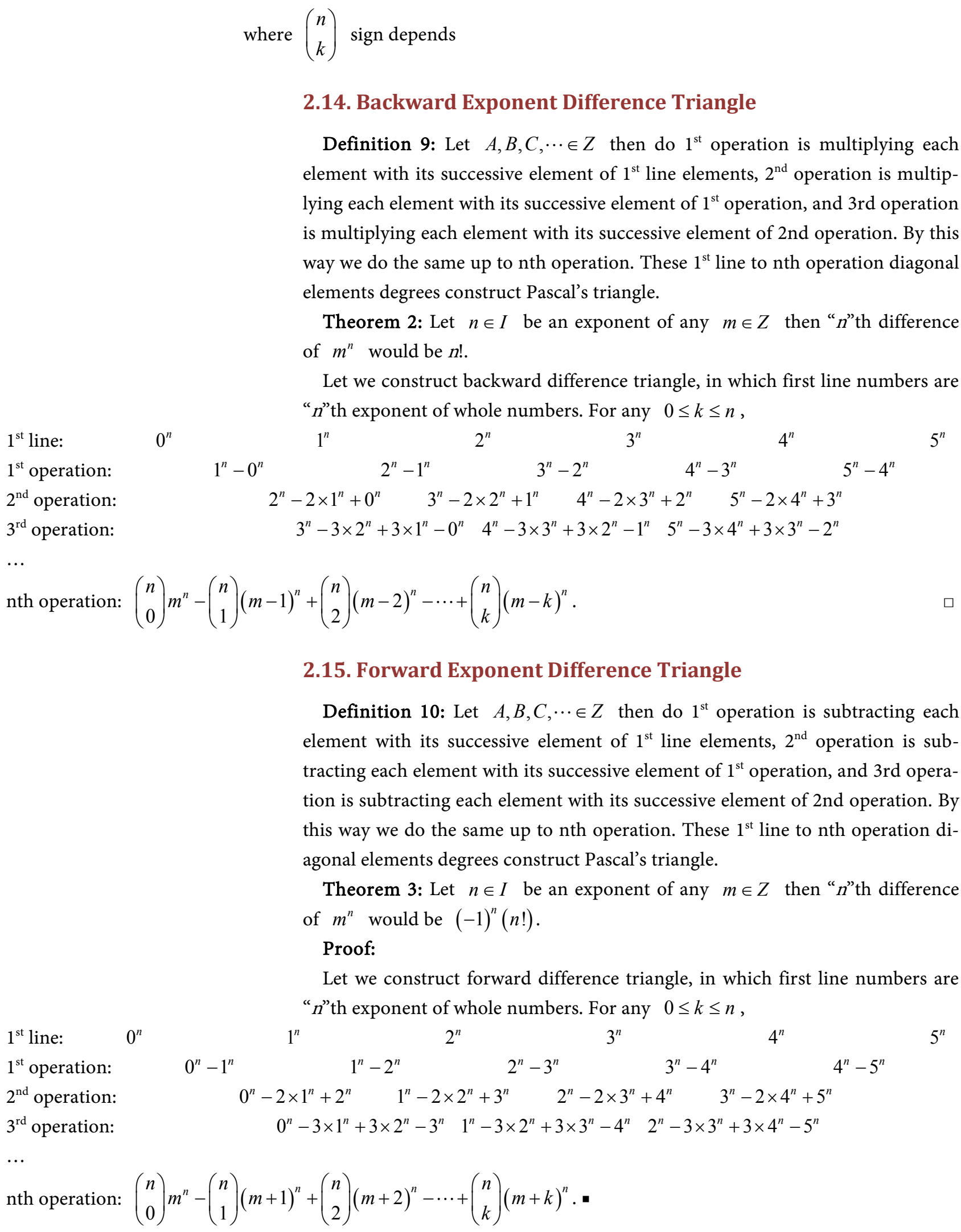

Examples for backward exponent difference method:

1) Let $m=0$ and $n=5$ then 


$$
\begin{aligned}
& 1 \times 0^{5}-5 \times(-1)^{5}+10 \times(-2)^{5}-10 \times(-3)^{5}+5 \times(-4)^{5}-(-5)^{5} \\
& =0+5-320+2430-5120+3125=120=5 !
\end{aligned}
$$

2) Let $m=-1$ and $n=5$ then

$$
\begin{aligned}
& 1 \times(-1)^{5}-5 \times(-2)^{5}+10(-3)^{5}-10 \times(-4)^{5}+5 \times(-5)^{5}-(-6)^{5} \\
& =-1+160-2430+10240-15625+7776=120=5 !
\end{aligned}
$$

3) Let $m=1$ and $n=5$ then

$$
\begin{aligned}
& 1 \times 1^{5}-5 \times 0^{5}+10 \times(-1)^{5}-10 \times(-2)^{5}+5 \times(-3)^{5}-(-4)^{5} \\
& =1-0-10+320-1215+1024=120=5 !
\end{aligned}
$$

Examples for backward exponent difference method:

1) Let $m=0$ and $n=4$ then

$$
\begin{aligned}
& 1 \times 0^{4}-4 \times 1^{4}+6 \times 2^{4}-4 \times 3^{4}+4^{4} \\
& =0-4+96-324+256=24=4 !
\end{aligned}
$$

2) Let $m=-1$ and $n=4$ then

$$
\begin{aligned}
& 1 \times(-1)^{4}-4 \times 0^{4}+6 \times 1^{4}-4 \times 2^{4}+3^{4} \\
& =1-0+6-64+81=24=4 !
\end{aligned}
$$

3) Let $m=1$ and $n=5$ then

$$
\begin{aligned}
& 1 \times 1^{5}-5 \times 2^{5}+10 \times 3^{5}-10 \times 4^{5}+5 \times 5^{5}-6^{5} \\
& =1-160+2430-10240+15625-7776=-120=-5 !
\end{aligned}
$$

\section{Conflicts of Interest}

The author declares no conflicts of interest regarding the publication of this paper.

\section{References}

[1] Pomerance, C. and Crandall, R. (2005) Prime Numbers: A Computational Perspective. 2nd Edition, Springer, New York.

[2] Chaudhuri, S. (2014) Fermat's Little Theorem-CS 2800: Discrete Structures.

[3] Balasubramani, P.R. (2019) Fermat's Theorem One Extension. Mathematical Sciences International Journal, 8, 6-10. 